\title{
Importancia económica y competitividad de las cadenas agropecuarias en Aguascalientes, México
}

\author{
Economic importance and competitiveness of agricultural chains \\ in Aguascalientes, Mexico
}

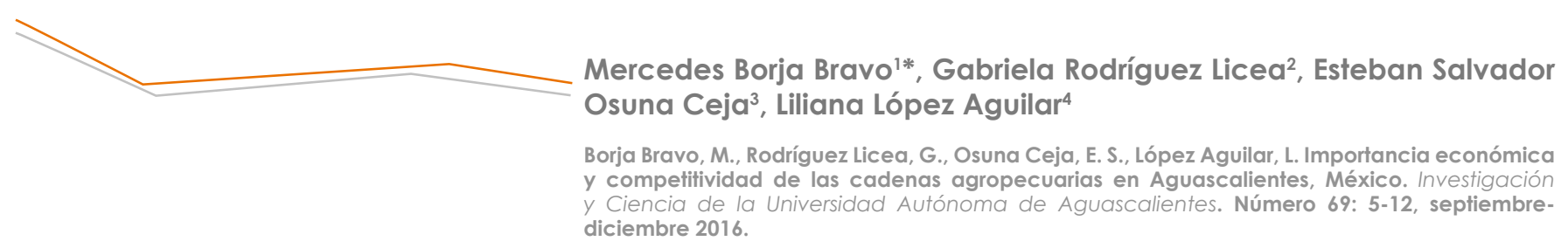

RESUMEN propiciada por las políticas públicas del estado, las cuales se enfocan en cadenas que requieran un menor consumo de agua y que generen productos

El presente trabajo tuvo por objetivo determinar la importancia socioeconómica y la competitividad de las cadenas agropecuarias en Aguascalientes, México. A través de la estandarización de indicadores y de la matriz de posicionamiento estratégico se encontró evidencia empírica de que, dada la integración vertical entre la producción agrícola y pecuaria, las cadenas prioritarias y estratégicas son las de maíz, bovinos, aves y porcinos. En contraste, las de frijol, uva, avena forrajera y pastos necesitan incrementar su importancia socioeconómica, mientras las de guayaba, durazno, nopal forrajero y alfalfa verde requieren impulsar la competitividad. Las hortalizas, ovinos, caprinos y miel, resultaron menos importantes y poco competitivas; sin embargo, muestran una tendencia expansiva

Palabras clave: estandarización, indicadores, posicionamiento, políticas, competitividad, importancia socioeconómica.

Keywords: standardization, indicators, positioning, policies, competitiveness, socioeconomic importance.

Recibido: 18 de agosto de 2015, aceptado: 30 de mayo de 2016

1 Programa de Socioeconomía, Instituto Nacional de Investigaciones Forestales, Agrícolas y Pecuarias Campo Experimental Pabellón.

2 Facultad de Economía, Universidad Autónoma del Estado de México.

3 Programa de Fertilidad de Suelos y Nutrición Vegetal, Instituto Nacional de Investigaciones Forestales, Agrícolas y Pecuarias Campo Experimental Pabellón.

4 Ingeniería en Desarrollo e Innovación Empresarial, Universidad Tecnológica del Norte de Aguascalientes.

* Autor para correspondencia: borja.mercedes@inifap.gob.mx con mayor oportunidad de posicionarse en el mercado, principalmente el del sector agroindustrial.

\section{ABSTRAC1}

The goal of this paper was to determine the socioeconomic importance and competitiveness of agricultural chains in Aguascalientes, Mexico. Through standardization of indicators and the array of strategic positioning empirical evidence is found that, given the vertical integration between the agricultural and livestock production, chains and strategic priority are maize, cattle, poultry and swine. In contrast, beans, grapes, forage oats and pastures, need to increase their socio-economic importance, and the guava, peach, prickly pear and green alfalfa forage, require boost competitiveness. The vegetables, sheep, goats and honey were less important and less competitive; however, they show an expansionary trend driven by the public policies of the state, which focus on strings that require a lower water consumption and generate products with greater opportunity to position itself in the market, mainly the agro-industrial sector.

\section{INTRODUCCIÓN}

En Aguascalientes, las actividades agropecuarias representan una fuente de empleo e ingresos para la población rural, son la base de la alimentación 
y tienen un efecto multiplicador sobre el sector agroindustrial. En 2013 representaron $69.3 \%$ de la superficie estatal y generaron 12,767.4 millones de pesos, de los cuales $80 \%$ representaron actividades pecuarias y $20 \%$ agrícolas. A pesar de lo anterior, la contribución del sector agropecuario del estado expresada en términos del Producto Interno Bruto (PIB) ha sido baja y decreciente, ya que pasó de $4.2 \%$ en 2003 a $3.8 \%$ en 2013; asimismo, el PIB agropecuario estatal solo contribuyó con $1.4 \%$ del PIB agropecuario nacional en 2013 y comparado con dicho indicador agropecuario de otros estados como Guanajuato, donde representó $31.4 \%$ para ese mismo año, es bajo (INEGI, 2013).

Entre los factores que explican la poca participación se encuentran la conservación de esquemas de producción y comercialización con poca tecnología, escaso financiamiento y alta dependencia climática, reflejados en la baja productividad y rentabilidad. Ante esto, las actuales políticas están impulsando la reactivación del campo y la modernización de la infraestructura a través de tres estrategias: uso eficiente del agua, incorporación de un patrón de cultivos apropiado para reducir la siniestralidad y mayor acceso a los mercados (Gobierno de Aguascalientes, 2011). Las acciones se han enfocado a proyectos agroalimentarios y de agregación de valor; no obstante, es necesario poner especial interés en las cadenas agroalimentarias con mayor impacto para el estado y potencializar el desempeño de aquellas que lo requieran.

Una cadena agroalimentaria se entiende como el proceso que sigue un producto agrícola o pecuario a través de las actividades de producción, transformación e intercambio hasta llegar a un consumidor final; en dicho proceso se incluye, también, el abasto de insumos y servicios de apoyo como son la investigación, capacitación y asistencia técnica. En México existen estudios sobre el análisis de la importancia socioeconómica y competitividad de las cadenas agroalimentarias estatales, tal es el caso de Aguirre Moreno (2010) y Sánchez Toledano et al. (2013), quienes identificaron las cadenas que requerían mayores incentivos para el desarrollo del sector agropecuario en Coahuila y Zacatecas, respectivamente. El análisis de las agrocadenas es un instrumento que permite diseñar y evaluar políticas públicas dirigidas a mejorar la competitividad, dado que a través de él se pueden planear estratégicamente políticas orientadas a fomentar la permanencia de las cadenas agroalimentarias exitosas e identificar las que requieren inversión e innovación tecnológica para ser competitivas (Sánchez Toledano et al., 2013). Es así como el objetivo de esta investigación fue, a partir de indicadores económicos y de competitividad, identificar y priorizar las necesidades en investigación y tecnología de las cadenas agropecuarias en Aguascalientes.

\section{MATERIALES Y MÉTODOS}

Dado que las cadenas agropecuarias están en función del producto primario (Gaitán et al., 2002), se aplicó el Método de Planificación del Desarrollo Tecnológico en Cadenas Agroindustriales propuesto por el Servicio Internacional para la Investigación Agrícola Nacional (Ghezán, 1999), el cual adopta el criterio de identificación de un producto genérico. Con base en lo realizado por Gaitán et al. (2002) y Aguirre Moreno (2010), se seleccionaron 11 cadenas agrícolas (maíz forrajero y grano, frijol, guayaba, avena forrajera, pastos, alfalfa verde, nopal forrajero, uva, durazno y hortalizas (lechuga, elote, chile verde, brócoli y ajo) y 7 pecuarias (aves carne, bovino leche, bovinos carne, porcinos, caprinos, ovinos y miel), las cuales se priorizaron con base en indicadores que miden: 1) la importancia socioeconómica; es decir, las características de las cadenas que las convierten en atributos importantes para ser consideradas como una actividad productiva pertinente (Sánchez Toledano et al., 2013) y 2) la competitividad, entendida como la capacidad dinámica de una cadena para mantenerse, ampliar y mejorar de manera continua y sostenida su participación en el mercado (Romero y Sepúlveda, 1999).

\section{Indicadores de la importancia socioeconómica}

Para analizar la importancia socioeconómica se consideraron los siguientes indicadores: a) Tamaño. Describe la dimensión de la cadena productiva en términos del valor de la producción y la contribución a la economía del estado; además de la aportación estatal en términos del volumen producido en el total nacional: [(valor de la producción estatal de la cadena)/(valor del sector agrícola o pecuario)]*100; y producción nacional: [(valor de la producción estatal de la cadena)/(valor de la producción nacional de la cadena)]*100; b) Dinamismo. Revela la dinámica de crecimiento del valor de la producción real y del volumen de producción, se mide con la 
tasa de crecimiento media anual (TCMA) del valor de la producción y volumen de la producción (2003-2013); c) Especialización. Muestra el grado de concentración y especialización económica regional de la cadena, se identifica si la cadena estatal destaca en términos comparativos con el nivel nacional: [(Participación del valor de la producción agrícola/pecuaria)/(Participación del valor de la producción del sector agropecuario nacional)]; y d) Cobertura social. Indica la participación porcentual que tiene el total de las unidades de producción del estado en la cadena: [(número de unidades productivas por cadena)/(unidades productivas del sector agrícola/pecuario)]*100 (Gaitán et al., 2002; Aguirre Moreno, 2010).

\section{Indicadores de la competitividad}

Para analizar la competitividad se consideraron los siguientes indicadores: a) Productividad. Describe los niveles de productividad, eficiencia en el uso los factores de producción y la dinámica del crecimiento estatal y nacional de la producción, está determinado por tres indicadores: 1) productividad relativa: [(rendimiento estatal por cadena)/(rendimiento nacional por cadena)]; 2) tasa relativa de crecimiento: [(TCMA estatal/TCMA nacional)]; y 3) el cambio en la aportación del estado a nivel nacional: [(aportación en la producción estatal, 2003-05)/(aportación en la producción estatal, 2011-13)]; b) Desempeño comercial. Permite comparar la evolución de los precios de los productores de la cadena con relación a la dinámica general y específica, se mide con los precios relativos al productor [(inflación de la cadena)/ (inflación del INPP)] y al consumidor [(inflación de la cadena/inflación del INPC)]; c) Sustentabilidad, está integrado por un grupo de indicadores cualitativos que resumen la evaluación de 19 expertos sobre la sustentabilidad ambiental de cada una de las cadenas; los temas considerados fueron la eficiencia en uso de agua, contaminación del suelo, aire y agua, y las prácticas de conservación y captación de agua; la evaluación se realizó aplicando una escala de medición alto-medio-bajo (Gaitán et al., 2002; Aguirre Moreno, 2010). Además, se midió la erosión del suelo, estimada a partir de la metodología propuesta por Martínez Ménez (2005). Cabe destacar que dada la falta de información para calcular la sustentabilidad, en las cadenas pecuarias no se consideró este indicador; y d) Integración. Es un indicador generado a partir de información proporcionada por los expertos a quienes se les preguntó sobre la participación del producto primario en la industria de la transformación y el mercado (cadenas comerciales y exportadores), las respuestas fueron evaluadas mediante una calificación de 1 a 10, a partir del promedio de estos dos criterios se calculó el indicador (Aguirre Moreno, 2010).

Una vez estimados los indicadores se estandarizaron y normalizaron; para ello, se asignó el valor más alto a la cadena con el mayor índice, una puntuación de uno a la cadena con el menor valor en el índice y se dividió la puntuación entre el valor máximo. Se utilizaron ponderadores que representan la contribución de cada criterio, los valores asignados se tomaron de Aguirre Moreno (2010): tamaño, 18\%; dinamismo, 33\%; especialización, 13\%; cobertura social, 36\%; productividad, 19\%; sustentabilidad, 26\%; desempeño comercial, $30 \%$; e integración, $25 \%$. Por último, se ubicó a cada cadena en cada cuadrante de la matriz de posicionamiento estratégico.

El volumen y valor de la producción, rendimiento y precio medio rural se obtuvieron del SIAP (2013); el número de unidades pecuarias y agrícolas por cultivo y especie de INEGI (2007) y el INNP e INPC de INEGI (2013). El periodo de análisis fue desde 2003 hasta 2013.

RESULTADOS

\section{Importancia socioeconómica}

De acuerdo a la Tabla 1, las cadenas de mayor tamaño (T) fueron las de guayaba, maíz forrajero, alfalfa, hortalizas, aves y bovinos leche, al tener una mayor aportación monetaria a la cadena agroalimentaria nacional y al valor de la producción estatal durante 2011-2013. En el dinamismo (D) destacaron las hortalizas, durazno, porcinos, aves y ovinos; las cuales mostraron el mayor crecimiento en volumen y valor de la producción. Las cadenas agrícolas con mayor especialización (E) fueron las de guayaba, nopal y maíz forrajero; mientras que las que no cuentan con especialización fueron las de maíz grano, frijol y pastos. La única cadena pecuaria especializada fue la de aves. Finalmente, se encontró que las cadenas con mayor cobertura social (CS) fueron las de maíz grano, frijol y bovinos leche y carne, ya que mostraron el mayor número de unidades de producción dedicadas a estas actividades. 
Tabla 1. Aguascalientes: Indicadores socioeconómicos de las cadenas agropecuarias

\begin{tabular}{|l|c|c|c|c|l|c|c|c|c|}
\hline \multicolumn{5}{|c|}{ Cadenas agrícolas } & \multicolumn{5}{c|}{ Cadenas pecuarias } \\
\hline Cadena & $\mathrm{T}$ & $\mathrm{D}$ & $\mathrm{E}$ & $\mathrm{CS}$ & Cadena & $\mathrm{T}$ & $\mathrm{D}$ & $\mathrm{E}$ & $\mathrm{CS}$ \\
\hline Maíz forrajero & 0.91 & 0.27 & 7.1 & 0.45 & Aves & 1.00 & 0.71 & 2.1 & 0.14 \\
\hline Maíz grano & 0.37 & 0.73 & 0.2 & 1.00 & Porcinos & 0.57 & 0.86 & 0.3 & 0.71 \\
\hline Frijol & 0.24 & 0.32 & 0.3 & 0.91 & Bovinos leche & 0.86 & 0.29 & 0.9 & 1.00 \\
\hline Guayaba & 0.96 & 0.36 & 22.2 & 0.73 & Bovinos carne & 0.71 & 1.00 & 0.3 & 0.86 \\
\hline Avena forrajera & 0.37 & 0.68 & 1.0 & 0.36 & Ovinos & 0.43 & 0.57 & 0.2 & 0.57 \\
\hline Pastos & 0.42 & 0.41 & 0.3 & 0.64 & Caprino & 0.14 & 0.14 & 0.1 & 0.43 \\
\hline Alfalfa verde & 0.69 & 0.27 & 1.6 & 0.82 & Miel & 0.29 & 0.43 & 0.2 & 0.29 \\
\hline Nopal forrajero & 0.51 & 0.36 & 12.8 & 0.55 & & & & & \\
\hline Uva & 0.46 & 0.68 & 6.4 & 0.18 & & & & & \\
\hline Durazno & 0.55 & 0.95 & 3.4 & 0.27 & & & & & \\
\hline Hortalizas & 0.60 & 0.95 & 1.2 & 0.09 & & & & & \\
\hline
\end{tabular}

Tabla 2. Aguascalientes: Indicadores de competitividad de las cadenas agropecuarias

\begin{tabular}{|l|c|c|c|c|l|c|c|c|}
\hline \multicolumn{5}{|c|}{ Cadenas agrícolas } & \multicolumn{5}{c|}{ Cadenas pecuarias } \\
\hline Cadena & $\mathrm{P}$ & $\mathrm{DC}$ & $\mathrm{S}$ & $\mathrm{I}$ & Cadena & $\mathrm{P}$ & $\mathrm{DC}$ & $\mathrm{I}$ \\
\hline Maíz forrajero & 0.33 & 2.12 & 0.45 & 0.45 & Aves & 0.67 & 0.26 & 0.86 \\
\hline Maíz grano & 0.67 & 2.34 & 0.45 & 0.36 & Bovino carne & 0.76 & 0.25 & 1.00 \\
\hline Frijol & 0.27 & 2.18 & 0.48 & 0.55 & Bovino leche & 0.62 & 0.34 & 0.71 \\
\hline Guayaba & 0.36 & 1.1 & 0.36 & 0.91 & Caprino & 0.76 & 0.17 & 0.14 \\
\hline Avena forrajera & 0.7 & 1.54 & 0.76 & 0.18 & Ovino & 0.52 & 0.19 & 0.29 \\
\hline Pastos & 0.58 & 1.6 & 0.94 & 0.09 & Porcino & 0.38 & 0.28 & 0.57 \\
\hline Alfalfa verde & 0.24 & 1.4 & 0.79 & 0.27 & Colmena & 0.29 & 0.3 & 0.43 \\
\hline Nopal forrajero & 0.36 & 0.93 & 0.67 & 0.64 & & & & \\
\hline Uva & 0.76 & 1.17 & 0.42 & 0.82 & & & & \\
\hline Durazno & 0.91 & 0.58 & 0.39 & 0.73 & & & & \\
\hline Hortalizas & 0.82 & 0.76 & 0.30 & 1.00 & & & & \\
\hline
\end{tabular}

\section{Competitividad}

Los resultados arrojaron que todas las cadenas agropecuarias (excepto frijol) fueron productivas (P) al registrar rendimientos por encima del promedio nacional. La uva, durazno, hortalizas, maíz grano, avena forrajera, aves, bovino carne, caprinos y ovinos tuvieron un crecimiento superior al de la cadena agropecuaria nacional. Las cadenas con mayor aportación de la producción nacional fueron las de uva, durazno, hortalizas, maíz grano y avena forrajera (Tabla 2). El mejor desempeño comercial (DC) lo registraron las cadenas de frijol y maíz grano y forrajero; es decir, que los precios al productor y consumidor mostraron una dinámica de crecimiento superior al de los precios nacionales; caso contrario ocurrió con el nopal forrajero, durazno, hortalizas y todas las cadenas pecuarias.

Las cadenas de pastos, alfalfa verde, avena y nopal forrajero mostraron mayor sustentabilidad (S); mientras que hortalizas, guayaba, uva, bovinos carne y leche y aves fueron las cadenas más integradas (I) a la agroindustria.

\section{Priorización de las cadenas agropecuarias}

En la matriz de priorización se puede identificar a las cadenas agropecuarias de prioridad estratégica para el estado con base en la importancia 


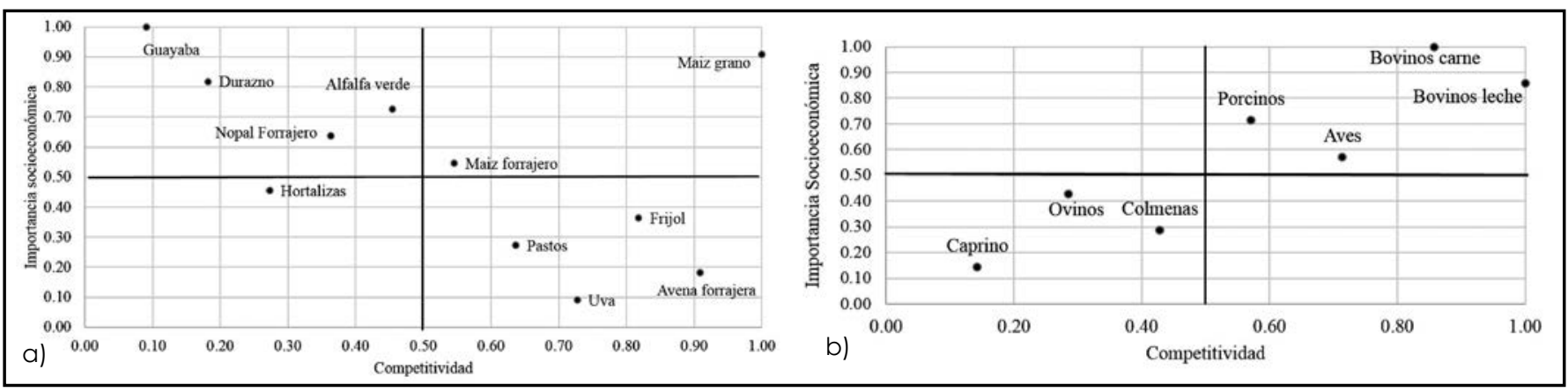

Figura 1. Aguascalientes: Matriz de priorización de las cadenas agropecuarias 2013. a) cadenas agrícolas, b) cadenas pecuarias. Elaboración propia con información obtenida en la estimación de los indicadores de las cadenas agrícolas y pecuarias.

socioeconómica y competitividad. Las cadenas de alta prioridad estratégica fueron las de maíz grano y forrajero, bovinos leche y carne, porcinos y aves (Figura 1).

\section{DISCUSIÓN}

Los indicadores muestran que existe una marcada integración entre las cadenas agrícolas y pecuarias (Figura 2), donde las primeras sostienen a las segundas, un ejemplo de ello son las cadenas de maíz forrajero y de bovinos carne y leche. El maíz forrajero es el principal insumo utilizado en la ganadería; no obstante, en los últimos diez años registró una pérdida en SU dinamismo y productividad; según datos del SIAP (2013), la producción de maíz forrajero disminuyó $-0.3 \%$ de 2003 a 2013 y tuvo un rendimiento menor al del promedio nacional. Existen varios factores que determinan el comportamiento de la producción de maíz forrajero, entre ellos, el elevado costo de semillas mejoradas. A lo anterior se suma el encarecimiento de los costos de producción por el incremento de los fertilizantes y menor disponibilidad de agua (Gobierno de Aguascalientes, 2011). Ante esta situación, es necesario el incremento en la generación y transferencia de tecnología que aumente la productividad del cultivo, basado en esquemas de conservación de suelo, mejor uso y aprovechamiento de agua y uso de variedades de alto rendimiento y tecnologías que permitan disminuir costos de producción.

Por su parte, el maíz grano es la cadena de mayor importancia social, representa una tradición productivo-cultural para los productores rurales y $56.5 \%$ de las unidades de producción de la entidad lo cultivan (INEGI, 2007); asimismo, el maíz grano mostró un buen desempeño comercial, ya que durante el lapso 2003-2013 los precios pagados al productor incrementaron a una tasa anual de 9.8\% (SIAP, 2013). Estas características de la cadena de maíz grano son similares a las reportadas por Sánchez Toledano et al. (2013) para el estado de Zacatecas, quienes determinaron que aun cuando existen limitantes en la productividad, especialización, alta siniestralidad en el cultivo y problemas de comercialización, la tendencia mundial a producir etanol ha provocado la reducción de la oferta y se han incrementado los precios. Para mantener esta actividad dentro de las principales cadenas estratégicas del estado es necesario implementar tecnologías que incentiven la productividad del cultivo bajo enfoques sustentables, principalmente en zonas de temporal, ya que $84 \%$ de la superficie destinada a este cultivo es bajo este sistema (SIAP, 2013).

En las cadenas de bovino carne y leche, porcinos y aves, se observó un bajo desempeño comercial provocado por los bajos precios pagados al productor, situación que es originada por las importaciones de carne y leche a menor precio que el interno y afecta el comportamiento de los precios en el mercado nacional (García Mata et al., 2004; Benítez Ramírez et al., 2010). Es así como la búsqueda de mejores precios para la carne y productos son un punto de atención inmediata que debe atenderse centrando la investigación en el otorgamiento de valor agregado a los productos derivados y en la búsqueda de nuevos mercados que ofrezcan mejores precios.

Las cadenas de frijol, avena forrajera, pastos y uva son consideradas como cadenas de impulso porque tienen poca importancia socioeconómica pero son competitivas. Al respecto, Sánchez Toledano 
et al. (2013) señalaron que estas cadenas podrían ser estratégicas en el desarrollo del sector agrícola, a través de políticas orientadas a incrementar los indicadores económicos y sociales.

La cadena de frijol fue la segunda más importante socialmente y en desempeño comercial; además, es un producto estratégico de la canasta bási$c a$, por lo que requiere mejorar su tamaño, dinamismo, especialización, productividad, sustentabilidad e integración. Específicamente, la transferencia de tecnología coadyuvaría a optimizar el aprovechamiento del agua, la conservación de suelos, control de plagas e incremento en el uso de variedades con alta preferencia por el consumidor, las cuales según Rodríguez Licea et al. (2010) son pinto, flor de mayo y flor de junio. En el caso de forrajes, la praticultura representa una alternativa sustentable, por lo que con el objetivo de lograr un mayor encadenamiento con las cadenas de carne y leche, la investigación se debe orientar a la introducción de gramíneas que sean compatibles con las condiciones agroclimatológicas.

La guayaba, durazno, alfalfa verde y nopal forrajero se ubicaron como cadenas de sostenimiento, caracterizadas por su alta importancia socioeconómica y baja competitividad. La guayaba es la cadena de mayor importancia socioeconómica para Aguascalientes, se caracteriza por ser la de mayor tamaño, especialización y con una importante cobertura social, además de ser una cadena productiva e integrada al mercado internacional y a la industria; sin embargo, demostró un bajo dinamismo, bajo desempeño comercial y poca sustentabilidad. A partir de la década de los noventa la cadena de guayaba comenzó a perder competitividad en el mercado nacional, lo que trajo como consecuencia que la participación de la producción estatal en el mercado nacional pasara de $38 \%$ en 2001 a 32\% en 2013 (SIAP, 2013). Según Rojas et al. (2000), los cambios tecnológicos son una alternativa para mejorar los índices de competitividad, razón por la cual la investigación se deberá centrar en introducir paquetes tecnológicos integrales que incrementen la productividad y permitan la generación de nuevas variedades que puedan cubrir el mercado fresco y agroindustrial a nivel nacional e internacional.

Una cadena de atención es la de nopal forrajero, ya que Aguascalientes aporta $23.61 \%$ de la producción nacional (SIAP, 2013). Esta cadena puede ser una alternativa en la producción de ganado, principalmente en los periodos de sequía prolongados; por tal motivo se requieren estrategias enfocadas a la expansión de la producción y a la investigación y transferencia de tecnología para la elaboración de dietas basadas en nopal. En el caso del durazno, el dinamismo en el crecimiento y la productividad lo posicionaron como cadena con potencial para convertirse en estratégica; no obstante, la poca cobertura social, los bajos precios en el mercado y la producción no sustentable son aspectos que deben subsanarse introduciendo tecnologías que contribuyan a optimizar el uso del agua. Es importante la investigación de mercados para identificar nichos de consumo de durazno fresco o con valor agregado insatisfechos en los que los productores tengan oportunidad de obtener mejores precios.

Finalmente, las hortalizas, miel, caprinos y ovinos se ubicaron como las cadenas con baja competitividad e importancia socioeconómica; las cuales además de ser consideradas como cadenas de mantenimiento para el estado, representan una alternativa para aprovechar los recursos y generar ingresos a los productores (Sánchez Toledano et al., 2013). Actualmente, en el estado se promueve la producción ovino-caprina, la cual demanda pocas cantidades de agua; sin embargo, los productores cuentan con poca experiencia en esta actividad, lo que representa una oportunidad para generar paquetes tecnológicos enfocados al manejo de estas especies.

Derivado de la productividad, especialización e integración, las hortalizas han registrado un importante crecimiento, se producen mayoritariamente a campo abierto; en 2013 la entidad solamente contaba con 87.1 ha de invernaderos (SEDRAE, 2013). Sin embargo, hoy día se está buscando diversificar la producción de hortalizas y los horticultores están explorando opciones de cultivos de mayor rentabilidad, como el espárrago. En atención a las nuevas tendencias, se debe impulsar el desarrollo tecnológico que cubra las necesidades de los cultivos recién explorados, al mismo tiempo se deben eficientizar los sistemas productivos de riego que ya existen a través de la adopción de tecnología propia para la agricultura protegida. 


\section{CONCLUSIONES}

El posicionamiento estratégico de las cadenas indicó que el maíz grano y forrajero dentro de la agricultura, bovinos de leche y de carne, aves y porcinos dentro del subsector pecuario son las cadenas prioritarias del estado. En contraste, las cadenas de frijol, uva, avena forrajera y pastos, requieren incrementar su importancia socioeconómica; mientras que las de guayaba, durazno, nopal forrajero y alfalfa verde, requieren incentivar la competitividad. Finalmente, las hortalizas, ovinos, caprinos y miel resultaron menos importantes para el estado y poco competitivas; sin embargo, muestran una tendencia expansiva propiciada por las políticas públicas del gobierno estatal, que se enfocan en la búsqueda de actividades agropecuarias que requieran un menor consumo de agua y generen productos con mayor oportunidad de posicionarse en el mercado, principalmente el del sector agroindustrial.

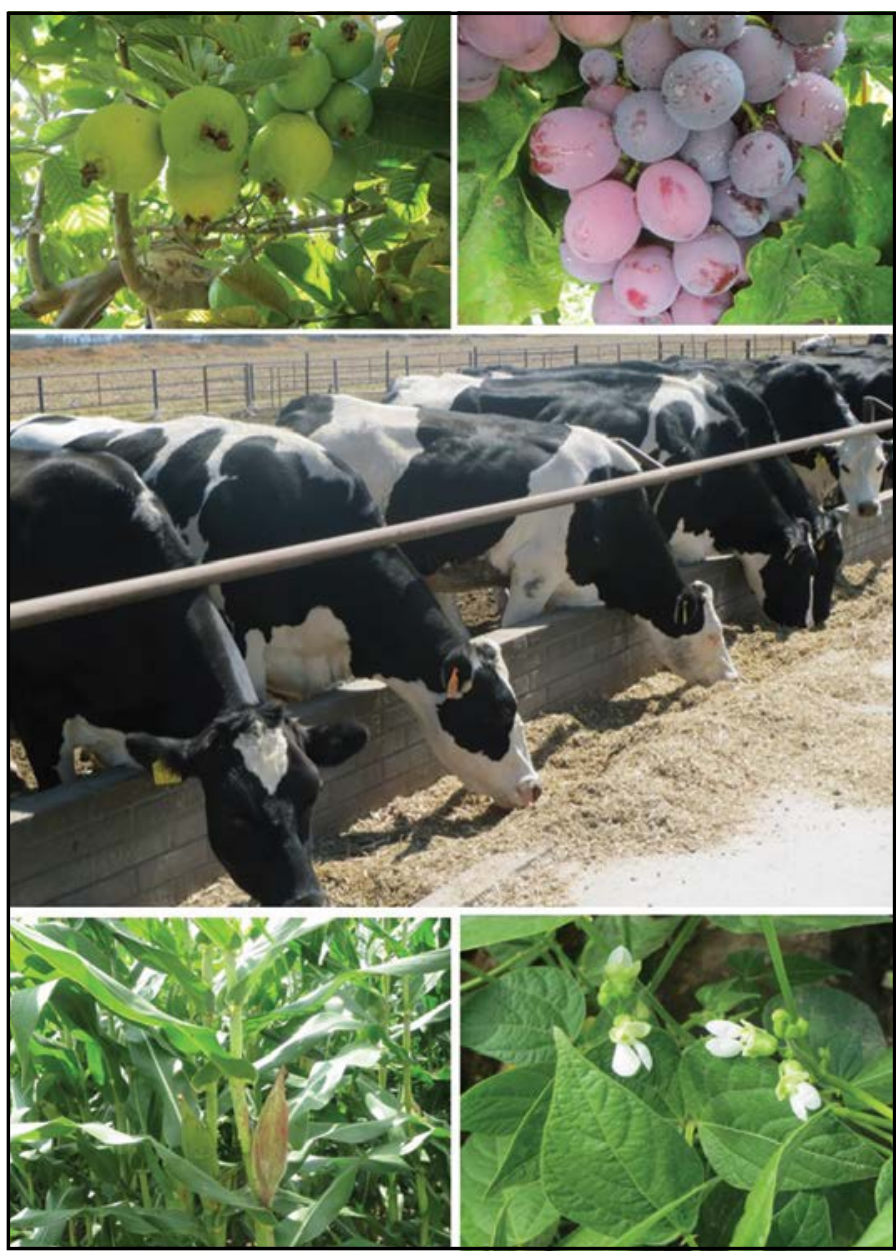

Figura 2. Cadenas agropecuarias de Aguascalientes. Fotografías tomadas por Mercedes Borja Bravo.
- AguirRe MORENO, V. J. et al. (Coords.). COAHUILA 2010. Diagnóstico de la problemática y oportunidades de desarrollo del sector rural de Coahuila. Coahuila, México: Universidad Autónoma Agraria Antonio Narro. 34 pp., 2010.

- BENÍTEZ RAMíREZ, J. G. et al. Determinación de los factores que afectan el mercado de carne bovina en México. Agrociencia, 44(1): 109-119, 2010.

- GAItÁN, J. et al. Programa estratégico de necesidades de investigación y transferencia de tecnología en Coahuila. Reporte Fase I: Recolección de información e identificación de cadenas productivas prioritarias. México: ITESM-COFUPROSAGARPA-Fundación Produce Coahuila, A. C. 93 pp., 2002.

- GARCÍA MATA, R. etal. Modelo econométrico para determinar los factores que afectan el mercado de la carne de porcino en México. Interciencia, 29(8): 414-420, 2004.
- GHeZÁN, G. et al. Análisis prospectivo de la demanda tecnológica en el sistema agroindustrial. La Haya, Países Bajos: ISNAR (SERVICIO INTERNACIONAL PARA LA INVESTIGACIÓN AGRÍCOLA NACIONAL), 1999.

- gobierno de AguASCAlientes. Programa Sectorial de Desarrollo Económico 2010-2016. Aguascalientes, México: Autor, 275 pp., 2011

- MARTínez MÉNEZ, M. Estimación de la erosión del suelo [Presentación en pdf]. México: SAGARPA (SECRETARÍA DE AGRICULTURA, GANADERÍA, DESARROLLO RURAL, PESCA Y ALIMENTACIÓN)-INCA RURAL-COP. 8 PP., 2005.

- RODRÍGUEZ LICEA, G. et al. Preferencias del consumidor de frijol (Phaseolus vulgaris L.) en México: factores y características que influyen en la decisión de compra diferenciada por tipo y variedad. Paradigma Económico, 2(1): 121-145, 2010. 
- ROJAs, P. et al. Algunos ejemplos de cómo medir la competitividad. Cuaderno Técnico No. 14. San José, Costa Rica: IICA. 49 pp., 2000.

- ROMERO, S. y SEPÚlVEDA, S. Territorio, agricultura y competitividad. Cuaderno Técnico No. 10. San José, Costa Rica: IICA. 11 pp., 1999.

- SÁNCHEZ TOLEDANO, B. I. et al. Estructura económica competitiva del sector agropecuario de Zacatecas: un análisis por agrocadenas. Revista Mexicana de Agronegocios, XVII(33): 552-563, 2013.

\section{De páginas electrónicas}

- INEGI (INSTITUTO NACIONAL DE ESTADÍStICA Y GEOGRAFÍA). Banco de información económica. México: Autor, 2013.
Recuperado en febrero de 2015, de http://www.inegi.org.mx/ sistemas/bie/

- INEGI (INSTITUTO NACIONAL DE ESTADÍStICA Y GEOGRAFÍA). Censo agrícola, ganadero y forestal 2007: resultados agrícolas por estado (Aguascalientes). México: Autor, 2007. Recuperado en abril de 2015, de http://www.inegi.org.mx/

- SEDRAE ISECRETARÍA DE DESARROLlO RURAL Y AGROEMPRESARIAL). Reporte de agricultura protegida. Aguascalientes, México. México: Autor, 2013. Recuperado en junio de 2015, de http://www.aguascalientes.gob.mx/sedrae/

- SIAP (SERVICIO DE INFORMACIÓN AGROALIMENTARIA Y PESQUERA). Sistema de Información Agroalimentaria de Consulta (SIACON), 1980-2013. Autor, 2013. Recuperado en abril de 2015, de http://www.siap.gob.mx/ asiacon2012parcialsiacon-zip/ 